but in the meantime the moral of the story is: avoid locums whenever possible.

R E Moshy Secretary, North-west Regional Hospital Junior Manchester M14 5RH

***The BMA Handbook for Hospital funior Doctors (obtainable from the BMA, £2.50) carries a warning to doctors "to consider very carefully the question of accepting locum appointments," and doctors are advised to seek the advice of the BMA before committing themselves to taking such posts.-ED, BMF.

\section{Guidelines on the performance of chemical pathology assays outside the laboratory}

SIR,-There is a growing concern among laboratory staff responsible for providing the routine analytical service from clinical biochemistry, clinical chemistry, or chemical pathology laboratories that the purchase of instruments for performing analytical work in wards and side rooms may not be always in the best interests of the patient.

The assays under consideration are at present mainly those of blood gases, acid base balance, and serum sodium, potassium, and paracetamol; but kits and apparatus for several other assays continue to be developed for use outside the main service laboratory. The siting of easy-to-use, automatic, pushbutton equipment in wards, clinics, and health centres provides the clinician with immediate results which could lead to earlier diagnosis or prompt adjustment of treatment. In favourable circumstances this may be achieved, but the proliferation of such equipment for use by personnel who may be untrained in laboratory practice, especially those aspects ensuring adequate quality control, and in criticism and assessment of the results, threatens the validity of assays and may introduce hazards for the patient.

Our aim in publishing this joint statement is to set out in general terms recommended procedures with the purpose of reducing discrepancies in performance of tests and of ensuring reliability and the highest quality of results and assays used in patient care. The possibility of inappropriate management of a patient arising from assay errors would thus be minimised. The widespread purchase of the aforementioned equipment together with the cost of manufactured materials could come from scientific budgets. Obviously every endeavour should be made to avoid duplication of expensive equipment and services by providing effective laboratory cover at all times. In exceptional circumstances, for geographical or other reasons, it may be necessary to carry out assays away from the main routine service laboratory. In that case we recommend that the following conditions should be met when performing chemical pathology assays outside the laboratory:

(1) Liaison-There should be full consultation and agreement with the head of the routine laboratory department before any equipment is purchased or obtained and close liaison should be maintained during its use.

(2) Choice of equipment-DHSS evaluations of equipment should be considered, as should local practice or equipment for similar assays carricd out in the routine laboratory. Selection, demonstration, and preliminary trials of equipment should be carried out in collaboration with the staff of the routine laboratory.

(3) Health and safety-The facilities provided and the equipment and techniques used should comply with the Health and Safety at Work Act and with the Code of Practice for the Prevention of Infection in Clinical Laboratories and Postmortem Rooms. There should be supervision by an appropriate safety officer. Advice should be obtained on the proper siting of the equipment.

(4) Training-No one should use analytical equipment without appropriate instruction or training in the operation of the equipment and in the concept of quality control. From time to time it may be necessary to retrain users. A formal list of trained users of equipment in side rooms and in small laboratories outside the main routine laboratory should be maintained and all those using the equipment should be aware of the medicolegal implications. Training in the use of the instrument and monitoring of subsequent performance should be supervised by a member of the staff of the routine laboratory. Records should be kept for each assay and its operator. Reports should be correctly prepared and filed in the patients' notes. They should be on a form recognisably different from the ordinary laboratory form. The system of units used should be agreed with the head of the routine laboratory.

(5) Quality control-Internal quality control should be performed at regular intervals and records kept and displayed as in the laboratory itself. An external quality assessment scheme should be used when practicable-for example, for potassium, sodium, etc. The routine laboratory quality control officer should be responsible to the head of the department for checking the records and initiating remedial action, etc, when necessary. A similar policy should apply to patient-operated apparatus so far as possible. The performance and the manner of performance should be checked at appropriate intervals, as agreed between the clinician and the staff of the routine service laboratory.

(6) Maintenance-The main routine laboratory should normally be responsible for $(a)$ periodic checking of simple day-to-day maintenance tasks carried out by the users; (b) the more complex maintenance, weekly or monthly (procedures involving dismantling of the equipment, cleaning or replacing parts); (c) dealing with simple breakdowns; $(d)$ service calls to the manufacturer. Patient-operated apparatus should be the responsibility of the relevant clinic. Laboratory support should be available for periodic checks on this type of equipment. Obviously routine service laboratory support is possible only if adequate information through consultation, as outlined in the above paragraphs, is available to the routine laboratory.

(7) Staffing-It may well be necessary to provide additional suitably qualified staff based on the routine service laboratory to meet these requirements. If possible the main operator of such equipment should be a member of the staff of the routine laboratory, which ensures continuity of methods of operation, maintains the service from a pool of the staff of the routine laboratory, and provides a career structure for the operator.

(8) General-It is essential that there should be full collaboration between medical and clinical staff outside the routine laboratory and the staff of the main routine laboratory, particularly in the supervision and training of personnel and the maintenance of equipment.

We would like to express our thanks to Drs Mary McMillan and John Cook for drafting these guidelines. President, Royal College of Pathologists London SW1Y 5AF President, Association of Clinical Pathologists London WXI ODN President, Association of Clinical Biochemist Birmingham $\mathrm{B5} 4 \mathrm{~J} \mathrm{~B}$

\section{A question of life and death}

SIR,-In the BBC2 debate "A question of life and death" on 19 February Dr Ronald Paul mentioned two patients who had fulfilled the British criteria of brain-stem death and who had survived. So fundamental are the implications of this claim that the basic facts must be made available to a very wide audience. May I therefore publicly-and through the courtesy of your columnsrequest the following information of Dr Paul ?

(1) The age and sex of the patients and the exact nature of the injuries from which they were suffering.

(2) The names of the surgeons who made the diagnoses of brain death-and the date at which the diagnoses were made. Was it before or after the publication, in November 1976, of the British criteria for the diagnosis of brain death ? Corroborative evidence from the surgeons who made the diagnoses would carry great conviction.

(3) The name of the laboratory to which the sera were sent for toxicological examination. The commonest cause, after all, of reversible apnoeic coma with absent brain-stem reflexes is drug intoxication.

(4) The electrolyte levels and temperature records in each case, to exclude the contributory role of metabolic disturbances or hypothermia.

(5) Whether cardiac or respiratory arrest at any stage complicated the evolution of these cases. It is well established-and stressed in the code-that if hypoxia has developed a second (delayed) examination is needed.

(6) The level to which the carbon dioxide tension rose during the 10-minute disconnection test or tests done to establish definitive apnoea.

(7) The final outcome in each of the mentioned cases.

(8) Whether Dr Paul is prepared to make the relevant documentation available to the Conference of Medical Royal Colleges for independent evaluation.

The requested information should be immediately available-or Dr Paul would surely not have mentioned with such confidence the cases in the middle of last week's debate.

Department of Medicine (Neurology),
Royal Postgraduate Medical School,
Hammersmith Hospital,

C Pallis

Hammersmith Hospital,

London W12 0HS

\section{Corrections}

Chlorosis, anaemia, and anorexia nervosa

We regret that in the letter by Dr Alan Lyell (7 February, $p$ 477) the date in the reference was given as 1926 instead of 1726 .

Training requirements for cosmetic surgery

In lines 9 and 10 of the letter by $\mathrm{Mr}$ Stewart Harrison (24 January, p 320) in the sentence beginning "This association, as $\mathrm{Mr} \mathrm{D} \mathrm{C} \mathrm{Herbert}$ and $\mathrm{Mr} \mathrm{N}$ G Buchan explain (22 November, $\mathrm{p}$ 1429), has been created ..." the reference to $\mathrm{Mr}$ Herbert and $\mathrm{Mr}$ Buchan was added in the course of subediting. Their association, however, is the British Association of Cosmetic Surgeons, whereas $\mathrm{Mr}$ Harrison is president of the Association of Cosmetic Plastic Surgeons. We apologise for any confusion that may have been caused. 